\title{
An Analysis of the Possible Significance of Music Education on the Practice of a Performer
}

\author{
Zijun Deng, a \\ ${ }^{1}$ University College London, Grower Street, London WC1E 6BT, UK \\ ${ }^{a}$ Emaile:dtnvzde@ucl.ac.uk
}

\begin{abstract}
The direct answer to whether we need music is yes, we do. The more relevant and pertinent question is why we need music. This paper will analyze the relevance of music to the human mind, human emotions, and human social life by focusing on psychological processes associated with musical stimuli.

The paper will delve into the roles of music psychology in the psychology field, and in related fields, in three parts:

- Auditory perception;

- Music cognition; and

- Music and musical impact on performance.

The paper will also seek to demystify these areas' relation to each other and specifically focus on the impact of these areas of music and music education on music learners and performers. It will also discuss how music education is significant in developing their capabilities in the creation of musical material and the adequate performance of the same, to establish the potential significance of the study of music education on the practice of a music performer.
\end{abstract}

Keywords: auditory perception, auditory reception, emotion, harmony, music, music cognition, music education, music perception, music psychology, perception.

\section{Introduction}

The analysis will focus on how music as a widespread cultural behavior influences music production; and, cognitive processes and attempt to illustrate the key points such as the development of temporary expectations within the confines of an experimental session and the planned or unplanned learning of new rhythmic sequences on music performance. It will further review the rhythmic benefit of a regular temporal cue on action memory and the personified and multimodal nature of cognition embedded in performers such as mature professional dancers' peripheral memory.

\section{OVERVIEW}

Music Psychology and music education in general, are vital in many sectors. The primary reasons for the study can be broken down into their impact on education, the related personnel, and society. These can be further classified by the characteristics of music on both the creators - the composers, producers, and the performers - as well as the audience - the reception, appreciation, and the effect of music and music education on them.
First, music is universally understood regardless of culture and language [19]. It is a vital non-verbal medium in connecting people across different cultural, social, and geographic divides because it can convey ideas and emotions, providing insight and understanding that transcends the spoken word. The most open age group to receiving new musical forms and ideas is statistically the teenagers, who believe that music blurs the boundaries between people of different age groups, economic standings, ethnic backgrounds, and social interests [2].

Music also improves memory retention skills that are vital for performers especially [11]. For example, this is evident in children who undergo musical training performing better in standardized tests. For example, a research study carried out at McMaster University by Dr. Fujioka [4] stated that "The children taking music lessons improved more over the year on general memory skills that are correlated with non-musical abilities, such as literacy, verbal memory, visuospatial processing, mathematics, and IQ, than did the children not taking lessons." The Scientific Journal Brain published this study indicating that young children with a year's worth of musical training had noticeable brain differences and 
improved memory compared to those without musical training. This translates to musical creators and performers who are generally far superior in their capabilities because of their ability to master numerous musical arrangements and to blend them, as they like to produce new unique creations that those with little or no music education cannot.

Music education develops perseverance and selfdiscipline for the performers [12]. When undertaken early in life, too, music education helps build the necessary stamina and skills such as circular breathing that are vital, especially for vocal performers to endure performing for extended periods while maintaining the quality of the performance [11].

Music education is vital for performers when playing instruments by building increased psychomotor skills and coordination and enabling the mental and physical capabilities to master and produce musical compositions effectively and perform them effectively.

According to Kraus [4], music fosters active listening skills; She conducted a study to investigate the impact of music education on the human brain. The results indicated that everyday listening skills are more robust in the musically-trained person than one without music training, which is evident in people who have just undergone sustained and rigorous music education. Furthermore, good listening skills directly influence the ability to discern speech within a noisy background, focus on it, and memorize it [19].

Music promotes teamwork, coordination, and collaboration amongst performers [8]. According to a Harris poll [8], "Seven in ten Americans (71\%) say that the learnings and habits from music education equip people to be better team players in their careers." Performers find it easier to work together in collaboration to create a typical composition. This is because they, having undergone music education, have a joint base upon which to build. They listen to one another, improvise and collaborate effectively because of the common grounding that they possess, which would not be possible without this grounding.

Music education is vital to the craft of professional dancers [9]. The rhythmic benefit of a regular temporal cue on action memory and the personified and multimodal nature of cognition embodied in their longterm memory enables them to perform efficiently by following the patterns that they learn.

Ultimately, music education enables performers to express themselves. This gives them the freedom to be creative and be both introspective and outgoing in creation, which provides both diversity and depth to their material.

\section{PRINCIPLES OF MUSIC PERCEPTION}

Music perception is the primary psychological factor that influences music performers [2]. The Perceptual principles in music are categorized and linked to one another, much like the guidelines to the perception of non-musical auditory materials, since no feature of human audition evolved specifically for music [2]. This does not mean that music is not unique, though, since acoustic signals in music have features specific to music in many cases [19]. The result is a proliferation of perceptual principles, which appear to characterize the music. These unique perceptual principles are perhaps why musicians are allowed to explore many different possibilities to utilize sounds; this is because the auditory perception principles generally relate to adaptation to the immediate environment and the deconstruction of gestalt principles [19].

These are the principles of characterizing music perception.

1) Systemic and chronological structures;

2) Ambiguity in the formation of the auditory stream formation; and,

3) Tonality formation.

\section{MUSIC AS A MICROSCOPE ON HUMAN LEARNING AND MEMORY}

The structure and perception of music give an insight into the learning and information retention processes because of human creative cognition, which is communicated as a component of an oral tradition [17]. This can be referenced in such scenarios as the best length of a melody for optimal learning and recall, which devices could help in remembrance, and the roles of rhythm and hierarchical organization in memory [17].

\section{EVERYDAY EMOTIONS AND MUSIC- RELATED EMOTIONS}

Music and emotion are related closely in many ways [17]. However, music-related emotions are difficult to pinpoint and describe whether they are common emotions experienced in everyday life. This problem comes full circle in attempting to address the bigger question: the actual definition of emotion and the necessity of analyzing the relation between music and emotion.

Music has adaptive value, and if the music does have evolutionary origins, it ought to mean that it relates to positive emotions [18]. However, the range of musicrelated emotions' broadness makes it improbable to convey music-related emotions and evolutionary adaptation alone. Therefore, the relation of music and 
emotion ought to investigate how emotion influences human evolution and cultures.

\section{MUSIC IN EVERYDAY LIFE}

In globalized Western folk theories, music's role is limited to hedonic listening, that is, for entertainment [5]. However, even in this limited context, it is acknowledged as having profound social implications, primarily because it is capable of modulating emotion; music exerts a significant influence on disparate areas. From the creation of self-identity to multimedia, music is diverse in different cultures. Many cultures view music as a participatory medium, supporting cognitive neuroscientific research, which has revealed extremely potent effects of participation on individuals' interaction and establishing a capability for empathic connection [5].

\section{THE INFLUENCE OF MUSIC ON INDIVIDUAL DEVELOPMENT}

\subsection{Learning and implementation of different values in music}

In their paper "Absolute pitch and relative pitch in music students: A comparison between East and West," Miyazaki et al. [15] conducted a test on music students in Japan, China, Poland, Germany, and the USA, in an attempt to determine their sensitivity to absolute pitch and relative pitch (AP and RP respectively).

The findings were that the Japanese students excelled in the Absolute Pitch test but struggled in the RP test. However, the Western participants had better results in the Relative Pitch test, but few had accurate AP. The most likely explanation for these AP and RP performance differences between the East and West reflects the differences in the music education practices and cultural settings that the students are exposed to [10]. These findings indicate that the development of AP perception and appreciation may affect the proper development of RP either directly or indirectly, that is, the development of one limits the development of the other. Bearing in mind that RP, not AP, is critically vital in music and music perception due to the relative arbitrariness of musical sounds, the current results reflect the problem with the underdevelopment of RP in the music education system currently in effect in Japan [12].

\subsection{Brain functions influenced by music.}

Previous studies have studied how various learning factors influence brain function while listening to and performing music, including singing activities. Still, none has uncovered the entire operation of the brain mechanism determined by musical learning, but what is clear is that music education influences brain mechanisms in some capacity (Maharaj, 2020).

\subsection{Implications from the bi-musical study}

According to Matsunaga et al. [16], the musical schema that the human brain acquires is culture-specific to the setting it develops within. In recent times, a new concept - bi-musicality - that represents musicality acquired through exposure to more than a single musical culture has come to the fore [16]. This implies that an individual can develop various schemata that gives the potential for more diversity to the performance.

\section{DIVERSITY AND HARMONY IN MUSIC}

The text version of a music composition/component is a set of sounds aimed at arranging the modal-rhythmic intonations aesthetically. This representation features sensual signals, cultural meanings, and the actualization of personal semantic experiences that create art emotions [17]. The mind looks for ways to complete the intrinsic world of work in any musical activity. This leads to various relations between human beings and music and the experience of a dynamic harmony of feeling and meaning in art appreciation [5]. Musical activity always relates to discovering musical harmony in the connection between sensuality, text, and purpose. The quest for this discovery happens in the sequence of text-sensualitymeaning-art emotion. The performer tries to find the harmony in the sequence text- meaning-artistic emotionsensuality, while the series that the composer follows is sensuality-artistic emotion-meaning-text [3].

\section{DIRECT EFFECTS OF MUSIC PSYCHOLOGY AND EDUCATION ON PERFORMANCE}

Musical education develops language and reasoning capabilities. Performers with a musical education background develop better language and brain function and better articulate their musical ideas, and a better creative prowess [6]. Musical psychology suggests that a firm musical foundation creates a mastery of memorization because even when using sheet music, performers are incessantly using memory to perform, and those with an excellent musical background have an easier time finding the correct arrangement to suit their performance structure.

Music develops psychomotor skills in many ways [9]. Playing the piano, striking a chord on the guitar, or achieving the appropriate finger configuration on the violin requires strong, well-coordinated fingers. Early training and training of fingers to be swift, nimble, and decisive early possesses long-term benefits to the prospective performers [21]. This early training enhances the ability to utilize small, precise muscle motions to write and develop other relevant skills, such as using a computer and performing other physical tasks. 
Performers can better develop Fine-tuned auditory skills in detecting and including meaningful, contentbearing elements in music, such as a gunshot in a song to tell a story about gun violence, or the emotion borne in a baby's cry, which provides a more somber feel to the composition. Performers who know about music psychology are better placed to pick out these sounds and make use of such cues in their music to enrich the experience, particularly recreational performers who end up being professionals [9].

The impact of music in a person's life begins at childhood since music builds imagination and intellectual curiosity in children [6]. Pursuing further development in musical proficiency serves to develop this creativity by fostering learning and curiosity. Like other forms of Artistic education, music psychology is critical in the development of imagination, the primary ingredient in music creation and performance [6].

The background education of music promotes discipline among those involved. For the content creators - the composers, singers, and instrumentalists, especially - because to learn, grow, and develop, they will need to be strict in their approach to learning and perfecting their craft. Though not focused on keenly, discipline is crucial in developing musical ability [1].

Musical education plays a key part in developing spatial intelligence, as people who study music tend to have superior spatial intelligence that aids their perception of the world and their ability to form mental pictures accurately [13]. This is especially important in developing performance sets and creating unique musical arrangements that seamlessly adapt to the environment in which it is performed, in both a physical, social, and cultural perspective.

Coordination amongst performers is more fluid if they have a grounding in music education since they are likely to have similar - or at least synchronous approaches to the creation of music and performance approach [9]. Therefore, the differences that may occur are bound to be primarily superficial ideas that stem from the individual's creative inclination. This is in no way a suggestion that music education makes similar artists; it just means that the necessary protocols that many music education programmes teach the students as part of a band or orchestra are ingrained in them, and this is the base upon which they build.

Musical education is a proponent for responsible risktaking amongst students who study music, primarily through alleviating their feelings of stage fright, a common problem amongst inexperienced performers [21]. By being exposed to the performance environment early, the performers learn to engage with this fear and anxiety, thereby improving the performer's selfconfidence, as well as encouraging the performer to take on environments that are more challenging to them, besides being willing to take content or sonic risks in their music, which enables them to scale new metaphorical heights, increasing their chances of success and attaining their potential [18].

\section{PERFORMERS' ACTIVE COGNITION OF THE EFFECTS OF MUSIC EDUCATION}

According to studies, performers who have a background in music education are more appreciative of the benefits of music education and recognize the importance of their musical experience in their craft (Hargreaves, 1986). Generally, the lessons are intrinsic and autonomous to the point where the performers do not have to pursue the knowledge actively, and therefore, they tend even to forget that it influences their performance. For example, stage use becomes autonomous or circular breathing when performing, which might affect such education lessons to be lost on the performers [7].

However, they recognize the benefits of the lessons in retrospect because such simple actions would not be so easy without a proper grounding in music. For example, John Legend famously told of how multiple talent searches rejected him [20]. Still, he understands why in retrospect because he had not been trained to perform as effectively as he does now. In this statement, he shows both the appreciation for the lack of musical education and its impact on the performer and the contrast that having a good grounding in musical education has on a performer [17]. Now, bearing in mind the career that John Legend has gone on to have after undergoing further training, establishing himself as one of the most influential singers of the past decade, the importance of musical education cannot be overstated.

The ideas posted here do not mean that performers with no training disregard its importance or that they are worse performers, nor does it mean that formal musical education is the only way to achieve proficiency in terms of performance. Instead, a performer's endeavours in teaching oneself to perform certain activities can be vital and enable the performer to appreciate the benefits even more. The result is that all performers, in one way or another, are appreciative of the role that a musical background plays in their performance proficiency.

\section{THE EFFECTS OF MUSIC EDUCATION ON AN AUDIENCE'S RECEPTION OF PERFORMED MUSICAL PIECES}

The audience is also affected by their musical background and their reception of performed musical pieces [1]. An audience will understand and appreciate a performance more if they have an amount of knowledge 
in music. The primary reason is that the audience will know what it takes to create a piece and therefore appreciate the complexities of the art, making them understand the performance more. For example, an audience made of members who have had experience in the music-making process will know how tough it is to create a piece and will appreciate the performer's efforts even more [18]. Conversely, the audience will also be more critical of an article, and any performance below par will be picked apart. Therefore, a knowledgeable audience will be more demanding of better performances. In turn, this will push the performers to better their craft and improve the execution of their performances [9].

An audience with little or no background knowledge of music, on the other hand, will, in most cases, accept the performance that is presented to them with no qualms. However, they might also not appreciate some performances because they do not understand what goes into making one or how difficult it is to produce the said piece. Moreover, most performance aspects might escape the audience because they might not understand the meanings of the different elements [19]. Therefore, they might think a piece to be wrong, below par, or pointless when there is no problem with the performance but preferably with their comprehension and appreciation of why each aspect of the performance is presented. Alternatively, an audience that is not educated well enough to understand them will, in most cases, not pick up intricate detail such as subtle instrumentals.

\section{MUSIC EDUCATION IN PERFORMANCE APPRENTICESHIP}

Music education is a vital tool in Performance Apprenticeship and helps both the mentor and the apprentice in many ways. For instance, a performer who has undergone musical training will find it easier to take on an apprentice and effectively mentor the apprentice because of the musical training that they experienced. In addition, the apprentice will find it easier to understand and implement the teachings if they have a musical background. The easiest way for the mentor; follow the template that got them there [11]. The presence of a predetermined teaching method will make it easier for teaching and learning. When conveyed from one person to another, the information will improve the apprentice and enable them to find new ways to enhance the conventions to evolve further and innovate new methods. It is only natural that many students will begin their musical journey with little to no prior knowledge and with an inability instantly connect with the traditional music literacy [11]. If the teacher or mentor has a background in musical education, they can use alternative methods to ease the teaching process. Introducing features such as color-coding, the use of icons, rhythm flash cards that are used to isolate individual sections in music, incorporating movement to teach rhythm or pitches, and using technology, with the overall result being more efficient mentorship.

\section{CONCLUSION}

As has been discussed, the study of music psychology and music education, in general, are pivotal in many ways, possessing a significant impact on education, the related personnel, and society. The consequences are particularly noteworthy because they affect music as a widespread cultural behaviour in terms of music production, performance, and the cognitive processes attached to musical audiences in terms of the development of temporary expectations within the confines of an experimental session and the learning of new rhythmic sequences. As has also been pointed out, music education (broadly conceived) is essential to the development of various aspects of the human mind, human emotions, and human social life because it influences musical stimuli' psychological processes.

Music education, particularly concerning music psychology, influences auditory perception, music cognition for both the performers and the audience, and the actual music and its impact on performance. Overall, the effect of music education serves the art form well, and as has been highlighted here, the more educated all the parties involved are, the better the probable outcome for both the audience and the performance because of the significance of the discussed study of music education on the practice of a music performer.

\section{REFERENCES}

[1] Behbehani, S., \& Steffens, J. (2020). Musical DIAMONDS: The influence of situational classes and characteristics on music listening behavior. Psychology of Music. https://doi.org/10.1177/0305735620968910

[2] Chi, Ching. (2020). Psychological Aspects of Musical Education. Manuskript. 241-245. 10.30853/manuscript.2020.10.44.

[3] Eaklor, V. L. (1985). Roots of an Ambivalent Culture: Music, Education, and Music Education in Antebellum America. Journal of Research in Music Education, 33(2), 8799. https://doi.org/10.2307/3344729

[4] Fujioka, T. (2006). Brain, Sept. 20, 2006; vol 129: pp 2593-2608. News release, Oxford University Press.

[5] Gaston, E. T. (1959). Book Review: The Social Psychology of Music. Journal of Research in Music Education, 7(1), 149150. https://doi.org/10.1177/002242945900700113 
[6] Giesbrecht, Maia. (2020). Musical Creativity and Music Education. 10.1163/9789004431409_005.

[7] Hargreaves, D. J. (1986). Developmental Psychology and Music Education. Psychology of Music, 14(2), 83-96. https://doi.org/10.1177/0305735686142001

[8] Harris, (2014). The Harris Poll ® \#72, July 24, 2014. Harris Poll and Public Relations Research.

[9] Hawkes, M. E. (2020). Experiences of developing pre-performance routines with recreational pianists. Psychology of Music. https://doi.org/10.1177/0305735620972788

[10] Herbst, A., de Wet, J., \& Rijsdijk, S. (2005). A Survey of Music Education in the Primary Schools of South Africa's Cape Peninsula. Journal of Research in Music Education, 53(3), 260283. https://doi.org/10.1177/002242940505300307

[11] Knyazeva, T.s. (2019). Musical Abilities and Intelligence as a Subject of Research in Music Psychology and Psychology of Music Education. Musical Art and Education. 7. 30-45. 10.31862/2309-1428-2019-7-3-30-45.

[12] Lee, Hyun-young. (2020). Music Education as Edification and Growth. Journal of Korean Philosophical Society. 155. 107-126. 10.20293/jokps.2020.155.107.

[13] Levi, D. S. (1978). Expressive Qualities in Music Perception and Music Education. Journal of Research in Music Education,26(4), 425435. https://doi.org/10.2307/3690713

[14] Maharaj, Adita \& Gill, Akini. (2020). Technology in Music Education.

[15] Miyazaki, Ken'Ichi \& Rakowski, Andrzej \& Makomaska, Sylwia \& Jiang, Cong \& Tsuzaki, Minoru \& Oxenham, Andrew \& Ellis, Gregory \& Lipscomb, Scott. (2018). Absolute Pitch and Relative Pitch in Music Students in the East and the West: Implications for Aural-Skills Education. Music Perception. $36 . \quad$ 135-155. 10.1525/mp.2018.36.2.135.

[16] Matsunaga, Rie; Hartono, Pitoyo; Abe, Jun-ichi. (2015). The acquisition process of musical tonal schema: Implications from connectionist modeling. Frontiers in Psychology, Vol 6, Sep 10 , 2015, Article 1348. https://psycnet.apa.org/doi/10.3389/fpsyg.2015.013 48

[17] Panda, Renato \& Malheiro, Ricardo \& Paiva, Rui Pedro. (2020). Audio Features for Music Emotion Recognition: a Survey. IEEE Transactions on
Affective Computing. PP. 1-1. 10.1109/TAFFC.2020.3032373.

[18] Peck, L. S. L. (2020). The 13th International Conference of Students of Systematic Musicology (SysMus20). Music \& Science. https://doi.org/10.1177/2059204320974215

[19] Pelayo III, Jose Maria. (2019). Music Psychology Journal.

[20] PTI, (2017). John Legend: I was rejected by every major record label. Times of India. https://timesofindia.indiatimes.com/entertainment/e nglish/music/news/John-Legend-I-was-rejected-byevery-major-recordlabel/articleshow/54713556.cms

[21] Rideout, R. R. (1982). On Early Applications of Psychology in Music Education. Journal of Research in Music Education,30(3), 141150. https://doi.org/10.2307/3345081 